\title{
ON HOP DOMINATION NUMBER OF SOME GENERALIZED GRAPH STRUCTURES
}

\author{
S. Shanmugavelan ${ }^{\dagger}, \quad$ C. Natarajan ${ }^{\dagger \dagger}$ \\ Srinivasa Ramanujan Centre, \\ SASTRA Deemed to be University, \\ Kumbakonam-612001, India \\ ${ }^{\dagger}$ shanmugavelan@src.sastra.edu, ${ }^{\dagger}$ natarajan_c@maths.sastra.edu
}

\begin{abstract}
A subset $H \subseteq V(G)$ of a graph $G$ is a hop dominating set (HDS) if for every $v \in(V \backslash H)$ there is at least one vertex $u \in H$ such that $d(u, v)=2$. The minimum cardinality of a hop dominating set of $G$ is called the hop domination number of $G$ and is denoted by $\gamma_{h}(G)$. In this paper, we compute the hop domination number for triangular and quadrilateral snakes. Also, we analyse the hop domination number of graph families such as generalized thorn path, generalized ciliates graphs, glued path graphs and generalized theta graphs.
\end{abstract}

Keywords: Hop domination number, Snake graphs, Theta graphs, Generalized thorn path.

\section{Introduction}

Domination in graphs is fascinating topic in the field of graph theory. It is one of the most effective mathematical models for a variety of real world problems. A simple undirected finite graph $G$ holds a vertex set $V(G)$ with vertices and an edge set $E(G)$ whose members are unordered pair of vertices called lines or edges of $G$. The degree of a vertex $v$, denoted by $d(v)$, is the number of edges that are incident with $v$ and the distance $d(u, v)$ between any two distinct vertices $u$ and $v$ is the length of the shortest path connecting $u$ and $v$ in $G$. We use the symbol $[n]=\{1,2, \ldots, n\}$. For any other graph theory terminology not defined here, we follow [3].

In a graph $G$, a subset $D \subseteq V(G)$ is said to be a dominating set if every vertex not in $D$ is adjacent to at least one vertex in $D$. The minimum cardinality of a minimal dominating set of $G$ is the domination number $\gamma(G)$. In the last three decades, several domination parameters have been established and they have been intensively investigated with applications in communication networks, facility location problems, game theory, mathematical chemistry, and so on. For a detailed study on domination concepts, one may refer [8-10].

Ayyasamy et al. [1] defined a new distance-based domination parameter called the hop domination number of a graph $G$. A subset $H \subseteq V(G)$ of a graph $G$ is a hop dominating set (HDS) if for every vertex $v$ not in $H$, there exists at least one vertex $u \in H$ such that $d(u, v)=2$. The minimum cardinality of a hop dominating set of $G$ is called the hop domination number of $G$ and is denoted by $\gamma_{h}(G)$. The hop degree of a vertex $v$ in a graph denoted by $d_{h}(v)$ is the number of vertices at distance $=2$ from $v$. The hop graph $H(G)$ of a graph $G$ is the graph having same vertex set and two vertices $u, v$ are adjacent in $H(G)$ iff $d_{G}(u, v)=2$. Also, Ayyasamy et al. [2] obtained some bounds on hop domination number for trees and characterized trees attaining those bounds. Natarajan et al. [13] found characterization results for hop domination number equals other domination parameters like total domination number, connected domination number for several families of graphs. Many scholars have explored this parameter in the years thereafter, leading to novel versions such as connected hop domination, total perfect hop domination, Roman hop domination, 
Global hop domination, etc., [11, 12, 15-18, 20]. In 2018, Natarajan et al. [14] discussed hop domination number for some special families of graph like central graph, middle graph and total graph. Recently, Packiavathi et al. [6] obtained the hop domination number of a caterpillar graph $P_{n}\left(l_{1}, l_{2}, \ldots, l_{n}\right)$ (a caterpillar is a graph obtained from the path by attaching leaves $l_{i}$ to $i^{\text {th }}$ vertex of the path $P_{n}$ ) and the domination number for some special families of snake graphs which occur as hop graph of $P_{n}(1,1, \ldots, 1)$ and $P_{n}(2,2, \ldots, 2)$. We refine their result on caterpillar graph and present an elegant result.

\section{Main results}

In this section, we study the hop domination number of snake graph families like triangular, alternate triangular, quadrilateral and alternate quadrilateral snakes. In addition, the hop domination number of some generalized structures like generalized theta graphs, generalized thorn paths and generalized ciliates graphs $G C(p, q, t)$ for $p=3$ and $p=4$ are determined.

Definition 1 [7]. Let $l_{1}, l_{2}, \ldots l_{n}$ be $n$ positive integers. Then the thorn graph $G^{t}=G^{t}\left(l_{1}, l_{2} \ldots l_{n}\right)$ is obtained from a graph $G$ by attaching $l_{i}$ pendant vertices (thorns) to each vertex $v_{i}$ of $G, i \in[n]$.

In 2020, Getchial Pon Packiavathi et al. [6] obtained the following result on caterpillar graphs.

Theorem 1 [6]. $\gamma_{h}\left(P_{n}(1,1, \ldots 1)\right)=\gamma_{h}\left(P_{n}(2,2, \ldots 2)\right)= \begin{cases}2 r, & \text { if } n=2 r \\ 2 r+3, & \text { if } \quad n=2 r+1 .\end{cases}$

First, we observe that the result given in Theorem 1 is wrong. For example, $\gamma_{h}\left(P_{4}(1,1,1,1)\right)=2$ whereas from their computations it is 4 . So, we refine the result by taking the more generalized version of caterpillar called thorn path $P_{n}^{t}$.

Theorem 2. For $n>1$,

$$
\gamma_{h}\left(P_{n}^{t}\right)= \begin{cases}\left\lfloor\frac{n-1}{2}\right\rfloor+1, & \text { if } n \equiv 0,1,3 \quad(\bmod 4) \\ \left\lceil\frac{n}{2}\right\rceil+1, & \text { if } n \equiv 2 \quad(\bmod 4) .\end{cases}
$$

P r o o f. Let $v_{1}, v_{2}, \cdots, v_{n}$ be the vertices of the central path $P_{n}$ in $P_{n}^{t}$ (see Fig. 1).

Case 1: $n \equiv 2(\bmod 4)$. In this case, any $\gamma_{h}$-set is of the form

$$
\left\{v_{i} \mid i \equiv 1 \quad(\bmod 4), 1 \leq i \leq(n-2)\right\} \cup\left\{v_{j} \mid j \equiv 2 \quad(\bmod 4), 2 \leq j \leq(n-1)\right\} \cup\left\{v_{n-1}\right\} .
$$

Thus,

$$
\gamma_{h}\left(P_{n}^{t}\right) \leq\left\lceil\frac{n}{2}\right\rceil+1
$$

and it is easily seen that

$$
\gamma_{h}\left(P_{n}^{t}\right) \geq\left\lceil\frac{n}{2}\right\rceil+1
$$

Therefore,

$$
\gamma_{h}\left(P_{n}^{t}\right)=\left\lceil\frac{n}{2}\right\rceil+1
$$

Case 2: $n \equiv 0,1,3(\bmod 4)$. In this case, any $\gamma_{h}$-set is of the form

$$
\left\{v_{i} \mid i \equiv 2 \quad(\bmod 4), 2 \leq i \leq(n-2)\right\} \cup\left\{v_{j} \mid j \equiv 3 \quad(\bmod 4), 3 \leq i \leq(n-1)\right\} \cup\left\{v_{n-1}\right\} .
$$




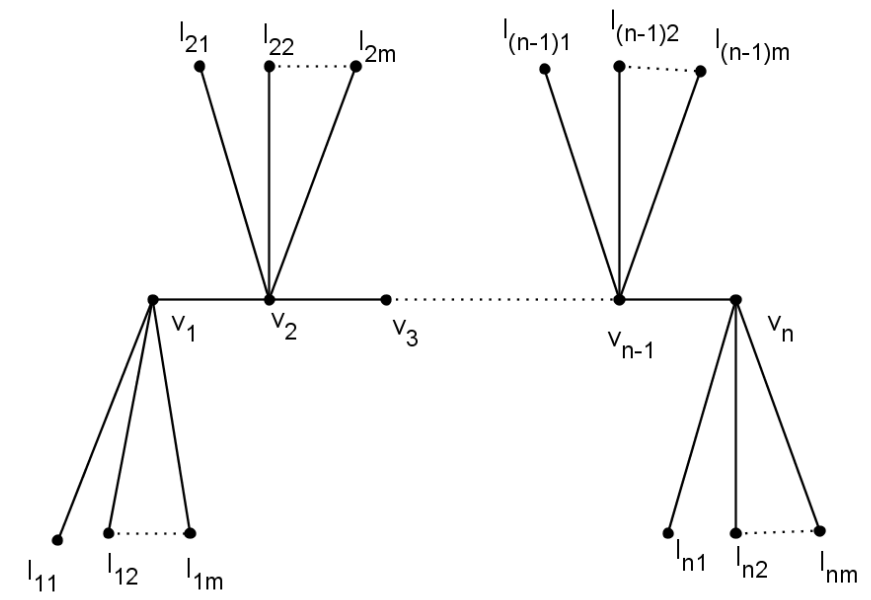

Figure 1. Thorny path $P_{n}^{t}$.

Thus,

$$
\gamma_{h}\left(P_{n}^{t}\right) \leq\left\lceil\frac{n}{2}\right\rceil
$$

and it is easily seen that

$$
\gamma_{h}\left(P_{n}^{t}\right) \geq\left\lceil\frac{n}{2}\right\rceil .
$$

Therefore,

$$
\gamma_{h}\left(P_{n}^{t}\right)=\left\lceil\frac{n}{2}\right\rceil .
$$

In [4], Derya Dogan et al. obtained some results for weak and strong domination in thorn graphs. Inspired by their results, we study our parameter namely, hop domination number for thorn rod given in [4], as well as for other generalized graph structures.

$$
\text { Lemma } 1 \text { [1]. } \gamma_{h}\left(P_{n}\right)= \begin{cases}2 r, & \text { if } n=6 r \\ 2 r+1, & \text { if } n=6 r+1 \\ 2 r+2, & \text { if } n=6 r+s, \quad 2 \leq s \leq 5 .\end{cases}
$$

Rewriting Lemma 1 in terms of congruence, we have

$$
\gamma_{h}\left(P_{n}\right)= \begin{cases}\left\lfloor\frac{n}{3}\right\rfloor, & \text { if } \quad n \equiv 0 \quad(\bmod 6) \\ \left\lfloor\frac{n}{3}\right\rfloor+1, & \text { if } \quad n \equiv 1,3,4,5 \quad(\bmod 6) \\ \left\lfloor\frac{n}{3}\right\rfloor+2, & \text { if } \quad n \equiv 2 \quad(\bmod 6) .\end{cases}
$$

Definition 2 [4]. A thorn rod is a graph $P_{n, t}$ which is obtained by taking a path on $n \geq 2$ vertices and attaching $(t-1)$ leaves, known as thorns, at each of the end of $P_{n}$. 


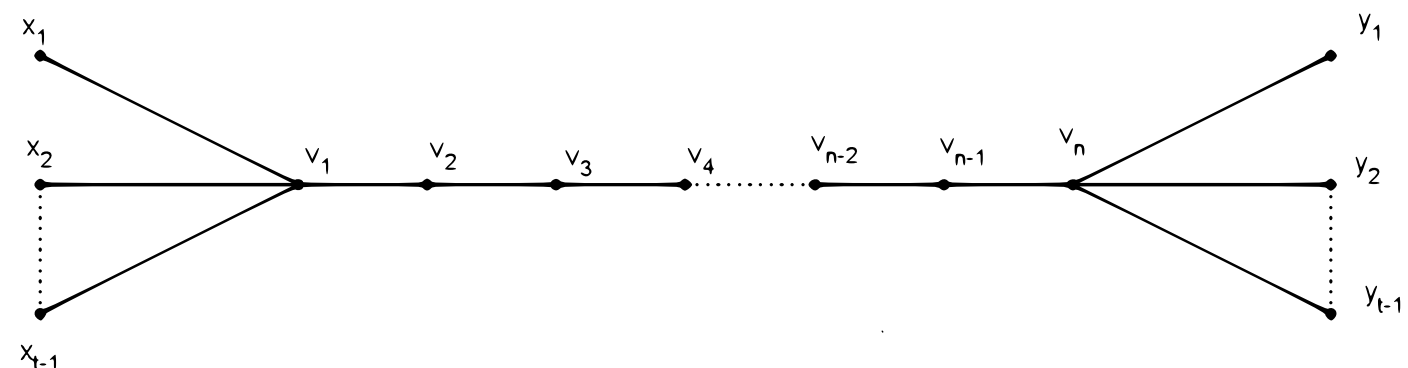

Figure 2. Thorn rod $P_{n, t}$.

Note that $P_{1, t}$ is a star graph $K_{1, t-1}$.

Theorem 3. $\gamma_{h}\left(P_{n, t}\right)= \begin{cases}\left\lfloor\frac{n-10}{3}\right\rfloor+6, & \text { if } n \equiv 0(\bmod 6) ; \\ \left\lfloor\frac{n-10}{3}\right\rfloor+5, & \text { if } n \equiv 1,2,3,5(\bmod 6) ; \\ \left\lfloor\frac{n-10}{3}\right\rfloor+4, & \text { if } n \equiv 4(\bmod 6) .\end{cases}$

$\mathrm{P}$ r o o f. Let us label the vertices of central path $P_{n}$ as $v_{1}, v_{2} \ldots v_{n}$. Let the leaves or thorns at the vertex $v_{1}$ be $x_{1}, x_{2}, \ldots x_{t-1}$ and the thorns at the vertex $v_{n}$ be $y_{1}, y_{2}, \ldots y_{t-1}$.

From Fig. 2, it is clear that to hop dominate $2(t-1)$ leaves and their support vertices, any $\gamma_{h}$-set must include the vertices $v_{2}, v_{3}, v_{n-1}, v_{n-2}$.

Now, the subgraph induced by $P_{n}-\left\{v_{1}, v_{2}, v_{3}, v_{4}, v_{5}, v_{n-4}, v_{n-3}, v_{n-2}, v_{n-1}, v_{n}\right\}$, is clearly a path on $(n-10)$ vertices.

By Lemma 1,

$$
\gamma_{h}\left(P_{n-10}\right)= \begin{cases}\left\lfloor\frac{n-10}{3}\right\rfloor, & \text { if } n \equiv 4 \quad(\bmod 6) ; \\ \left\lfloor\frac{n-10}{3}\right\rfloor+1, & \text { if } n \equiv 0,1,3,5 \quad(\bmod 6) \\ \left\lfloor\frac{n-10}{3}\right\rfloor+2, & \text { if } n \equiv 2 \quad(\bmod 6) .\end{cases}
$$

and hence $\gamma_{h}(G)=4+\gamma_{h}\left(P_{n-10}\right)$. Thus, the result follows.

Definition 3. A glued path $G P(n, t)$ is a graph obtained by gluing $t$ copies of a path $P_{n}(n \geq 2)$ at a common vertex $v$ such that $v$ is the initial vertex in each copy of $P_{n}$.

Theorem 4. $\gamma_{h}(G P(n, t))= \begin{cases}2\left\lceil\frac{n}{6}\right] t, & \text { if } n \equiv 0,5(\bmod 6) ; \\ 2\left\lfloor\frac{n}{6} \mid t+1,\right. & \text { if } n \equiv 1(\bmod 6) ; \\ 2\left[\left\lceil\frac{n}{6}\right\rceil t-1\right]+1, & \text { if } n \equiv 4(\bmod 6) ; \\ 2\left\lfloor\frac{n}{6} \mid t+2,\right. & \text { if } n \equiv 2,3(\bmod 6) .\end{cases}$

P r o o f. Let us arrange the vertices of $G P(n, t)$ row-wise subject to the following conditions:

(i) Place the common vertex in the $1^{\text {st }}$ row $R_{1}$.

(ii) First vertex of each copy of the path $P_{n}$ be placed in the $2^{\text {nd }}$ row $R_{2}$. 


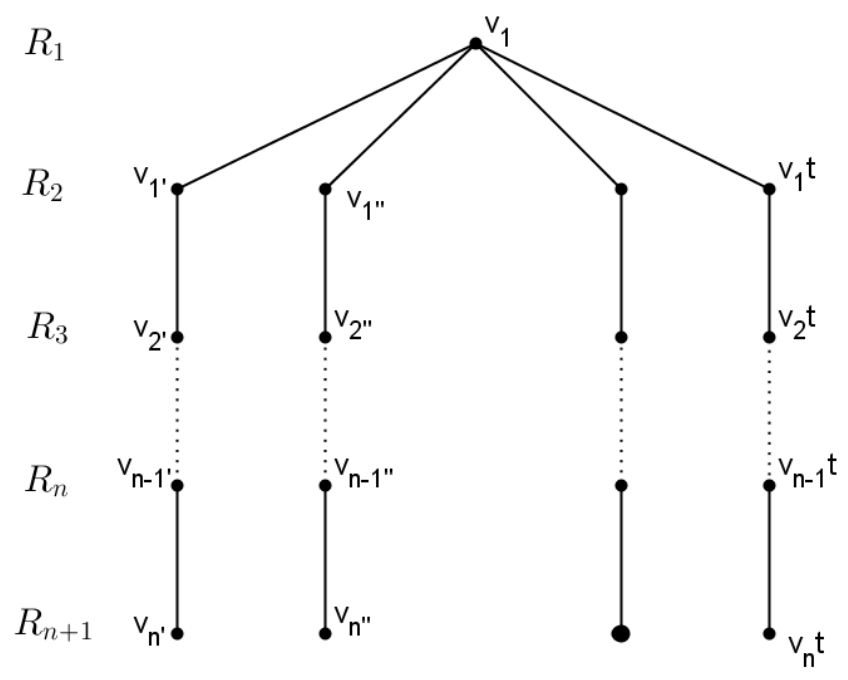

Figure 3. Glued path $G P(n, t)$.

(iii) In general, $n^{\text {th }}$ vertex of each copy in the $(n+1)^{t h}$ row $R_{n+1}$.

From Fig. 3, it is clear that, each row $R_{i}$ has $t$ vertices except the first row. That is, $V\left(R_{i}\right)=$ $\left\{v_{i}, v_{i}^{\prime \prime}, \ldots, v_{i}^{(t)}\right\}, 2 \leq i \leq(n+1)$. To hop dominate all those leaves and support vertices, all the vertices in $R_{n-1}$ and $R_{n-2}$ must be selected from a $\gamma_{h}$-set of $G P(n, t)$. This choice will also hop dominate all of the vertices of $R_{n-3}$ and $R_{n-4}$.

Case 1: $n \equiv 0(\bmod 6)$. In this case, any $\gamma_{h}$-set contains $2\lceil n / 6\rceil t$ vertices from the following rows $\mathcal{R}=\left\{R_{(n-1)}, R_{(n-2)}, R_{(n-7)}, R_{(n-8)}, \ldots, R_{4}, R_{3}\right\}$. Thus,

$$
\gamma_{h}(G P(n, t)) \leq 2\left\lceil\frac{n}{6}\right\rceil t .
$$

It is easy to observe that any hop dominating set of $G P(n, t)$ contains at least $2\lceil n / 6\rceil t$ vertices. Therefore,

$$
\gamma_{h}(G P(n, t))=2\left\lceil\frac{n}{6}\right\rceil t .
$$

Case 2: $n \equiv 5(\bmod 6)$. In this case, any $\gamma_{h}$-set includes $2\lceil n / 6\rceil t$ vertices from the rows $\left(\mathcal{R} \backslash R_{3}\right) \cup\left\{v_{1}\right\}$. Thus,

$$
\gamma_{h}(G P(n, t)) \leq 2\left\lceil\frac{n}{6}\right\rceil t
$$

Also,

$$
\gamma_{h}(G P(n, t)) \geq 2\left\lceil\frac{n}{6}\right\rceil t
$$

Therefore,

$$
\gamma_{h}(G P(n, t))=2\left\lceil\frac{n}{6}\right\rceil t .
$$

Case 3: $n \equiv 1(\bmod 6)$. In this case, any $\gamma_{h}$-set includes vertices from the following rows $\mathcal{R}^{\prime}=\left\{R_{(n-1)}, R_{(n-2)}, R_{(n-7)}, R_{(n-8)}, \ldots, R_{5}, R_{4}\right\} \cup\left\{v_{1}\right\}$. Thus,

$$
\gamma_{h}(G P(n, t)) \leq 2\left\lfloor\frac{n}{6}\right\rfloor t+1 .
$$


One can observe that

$$
\gamma_{h}(G P(n, t)) \geq 2\left\lfloor\frac{n}{6}\right\rfloor t+1
$$

Therefore,

$$
\gamma_{h}(G P(n, t))=2\left\lfloor\frac{n}{6}\right\rfloor t+1 .
$$

Similarly, the proof follows for other cases.

The generalized thorn path can be defined as follows,

Definition 4. The graph obtained by taking a path $P_{n}$ and attaching $t$ copies of $P_{r}$ to every vertex of $P_{n}$ is said to be a generalized thorn path and denoted by $G(n, r, t), n>1$.

Theorem 5. $\gamma_{h}[G(n, r, t)]= \begin{cases}\gamma_{h}\left(P_{n}\right)+n t\left\lfloor\frac{r}{3}\right\rfloor, & \text { if } \quad r \equiv 0(\bmod 6) ; \\ n+n t\left\lfloor\frac{r}{3}\right\rfloor, & \text { if } \quad r \equiv 1,2,3(\bmod 6) ; \\ n t\left(\left\lfloor\frac{r}{3}\right\rfloor+1\right), & \text { if } \quad r \equiv 4,5(\bmod 6) .\end{cases}$

P r o o f. Let $S=\left\{v_{i j}^{\prime}: 1 \leq i \leq r, 1 \leq j \leq t\right\}$ denote the vertices of the $i^{t h}$ copy of $P_{r}$ as shown in Fig. 4.

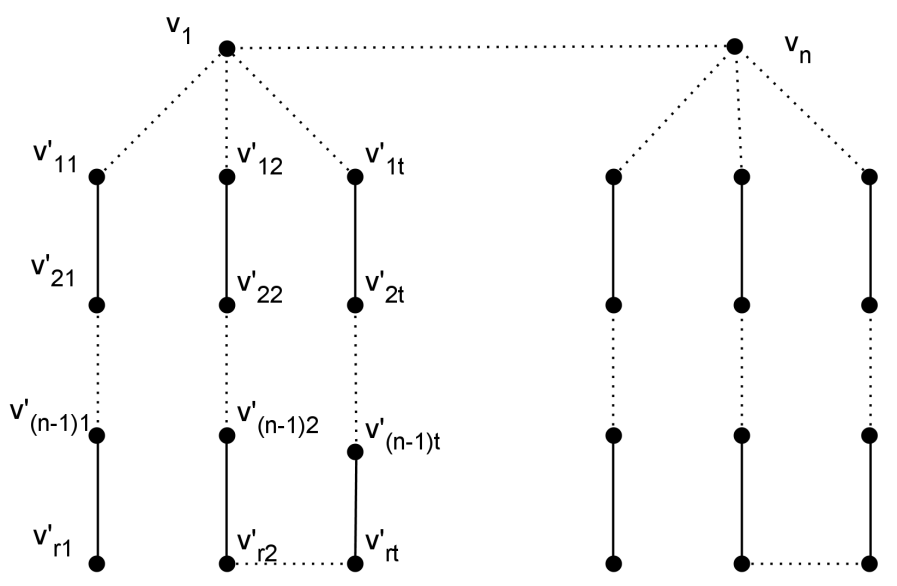

Figure 4. Generalized thorn path $G(n, r, t)$.

Case 1: $r \equiv 0(\bmod 6)$. In this case, the set

$$
H^{\prime}=\left\{v_{(n-2) j}^{\prime}, v_{(n-1) j}^{\prime}, v_{(n-8) j}^{\prime}, v_{(n-9) j}^{\prime} \ldots v_{4 j}^{\prime}, v_{3 j}^{\prime}, 1 \leq j \leq t\right\}
$$

hop dominates all vertices in each copy of $P_{r}$. In order to hop dominate the vertices of the path $P_{n}$, any $\gamma_{h}$-set of $G(n, r, t)$ should contains $\gamma_{h}\left(P_{n}\right)$ vertices. As a result,

$$
\gamma_{h}[G(n, r, t)] \leq \gamma_{h}\left(P_{n}\right)+\left|H^{\prime}\right|=\gamma_{h}\left(P_{n}\right)+n t\left\lfloor\frac{r}{3}\right\rfloor .
$$

It is easily seen that

$$
\gamma_{h}[G(n, r, t)] \geq \gamma_{h}\left(P_{n}\right)+\left|H^{\prime}\right|=\gamma_{h}\left(P_{n}\right)+n t\left\lfloor\frac{r}{3}\right\rfloor .
$$


Therefore,

$$
\gamma_{h}[G(n, r, t)]=\gamma_{h}\left(P_{n}\right)+\left|H^{\prime}\right|=\gamma_{h}\left(P_{n}\right)+n t\left\lfloor\frac{r}{3}\right\rfloor .
$$

Case 2: $r \equiv 1,2,3(\bmod 6)$. Here, any $\gamma_{h^{-}}$set contains the set

$$
H^{\prime}=\left\{v_{(n-2) j}^{\prime}, v_{(n-3) j}^{\prime}, v_{(n-8) j}^{\prime}, v_{(n-9) j}^{\prime} \ldots v_{2 j}^{\prime}, v_{1 j}^{\prime}, 1 \leq j \leq t\right\}
$$

and so $H^{\prime} \cup V\left(P_{n}\right)$ forms a $\gamma_{h}$-set of cardinality $n+\left|H^{\prime}\right|$.

Case 3: $r \equiv 4,5(\bmod 6)$. In this case, vertices in attached $t$ copies are sufficient for a $\gamma_{h}$-set of $G(n, r, t)$. Therefore,

$$
\gamma_{h}[G(n, r, t)]=n t \gamma_{h}\left(P_{r}\right) \leq n t\left(\left\lfloor\frac{r}{3}\right\rfloor+1\right) .
$$

Also, any minimal HDS of $G(n, r, t)$ requires at least

$$
\gamma_{h}[G(n, r, t)]=n t \gamma_{h}\left(P_{r}\right) \geq n t\left(\left\lfloor\frac{r}{3}\right\rfloor+1\right)
$$

vertices. Hence,

$$
\gamma_{h}[G(n, r, t)]=n t \gamma_{h}\left(P_{r}\right)=n t\left(\left\lfloor\frac{r}{3}\right\rfloor+1\right) .
$$

Definition 5 [19]. A generalized theta graph $\theta[n P(m)]$ is a graph obtained from $n$-internally disjoint paths, in which each path $P(m)$ contains $m$ internal vertices and these paths share common end vertices $u$ and $v$ (see Fig. 5).

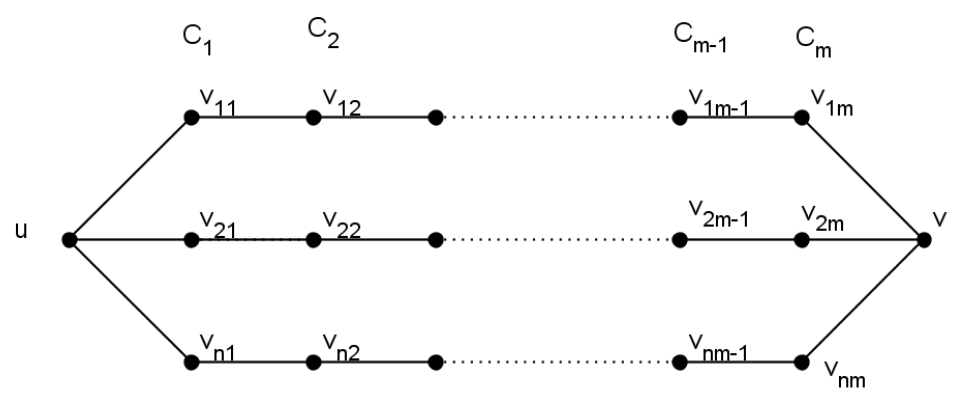

Figure 5. Generalized theta graph $\theta[n P(m)]$.

Theorem 6. $\gamma_{h}(\theta[n P(m)])= \begin{cases}4+\left\lfloor\frac{m-6}{3}\right\rfloor, & \text { if } \quad m \equiv 0(\bmod 6) ; \\ 4+n\left[\left\lfloor\frac{m-6}{3}\right\rfloor+1\right], & \text { if } \quad m \equiv 1,3,4,5(\bmod 6) ; \\ 4+n\left[\left\lfloor\frac{m-6}{3}\right\rfloor+2\right], & \text { if } \quad m \equiv 2(\bmod 6) .\end{cases}$

P r o o f. Let us denote $\theta[n P(m)]$ by $G$ for convenience. Clearly, $\{u, v\}$ should be included in any $\gamma_{h}$-set and any one vertex from column $C_{1}$ and $C_{m}$ is enough to hop dominate the vertices in $C_{1}$ and $C_{m}$. The induced subgraph $\left\langle G-\{u, v\} \cup C_{1} \cup C_{2} \cup C_{m-1} \cup C_{m}\right\rangle$ is a collection of n-distinct paths $P_{m-4}$. As a consequence of Lemma 1 , the result follows. 


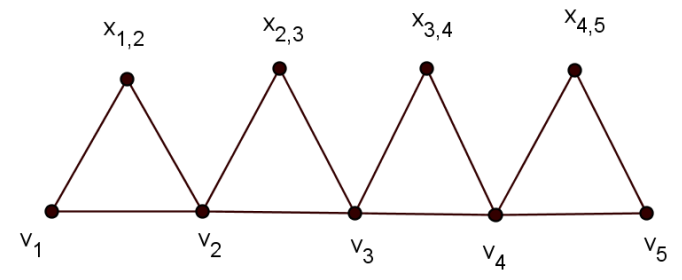

Figure 6. Triangular snake $T_{4}$.

Definition 6 [5]. A triangular snake graph $T_{n}$ is a graph obtained from the path $P_{n}$ by replacing each edge by a cycle of length 3 . For example, a triangular snake $T_{4}$ is shown in Fig. 6

A double triangular snake $D T_{n}$ consists of two triangular snakes that have a common path.

That is, a double triangular snake is obtained from a path $v_{1}, v_{2}, \ldots, v_{n}$ by joining $v_{i}$ and $v_{i+1}$ to a new vertex $x_{i}$ for $i=1,2, \ldots, n-1$ and to a new vertex $y_{i}$ for $i=1,2, \ldots, n-1$. For example, a double triangular snake $D T_{6}$ is illustrated in Fig. $\%$.

A triple triangular snake $T T_{n}$ is a graph in which three triangular snakes have a common path. Similarly, a four triangular snake $F T_{n}$ is a graph in which four triangles share a common path.

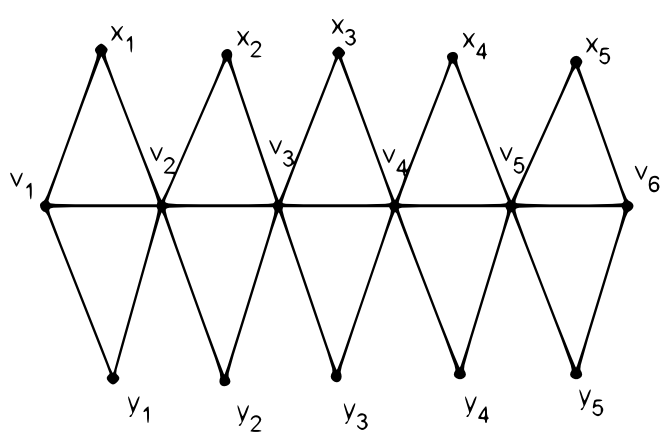

Figure 7. Double triangular snake $D T_{6}$.

Remark 1. $\gamma_{h}\left(T_{n}\right) \geq 3$.

\section{Theorem 7 .}

$$
\gamma_{h}\left(T_{n}\right)=\gamma_{h}\left(D T_{n}\right)=\gamma_{h}\left(T T_{n}\right)=\gamma_{h}\left(F T_{n}\right)= \begin{cases}2+\left\lfloor\frac{n-3}{2}\right\rfloor, & \text { if } n \text { is odd }(n \neq 3), \\ 2+\left\lfloor\frac{n-2}{2}\right\rfloor, & \text { if } n \text { is even }(n \neq 6) .\end{cases}
$$

P r o o f. First, we observe that any $\gamma_{h}$-set of $T_{n}$ will also be a $\gamma_{h}$-set for $D T_{n}, T T_{n}$ and $F T_{n}$ because they share a common path.

For $n=3$ and $6, \gamma_{h}\left(T_{3}\right)=\gamma_{h}\left(T_{6}\right)=3$.

Let us label the vertices of the common path as $\left\{v_{1}, v_{2} \ldots v_{n}\right\}$ and the remaining vertices of $T_{n}$ be $S=\left\{x_{i, i+1}\right\}, 1 \leq i \leq(n-1)$ as shown in Fig. 8 . 


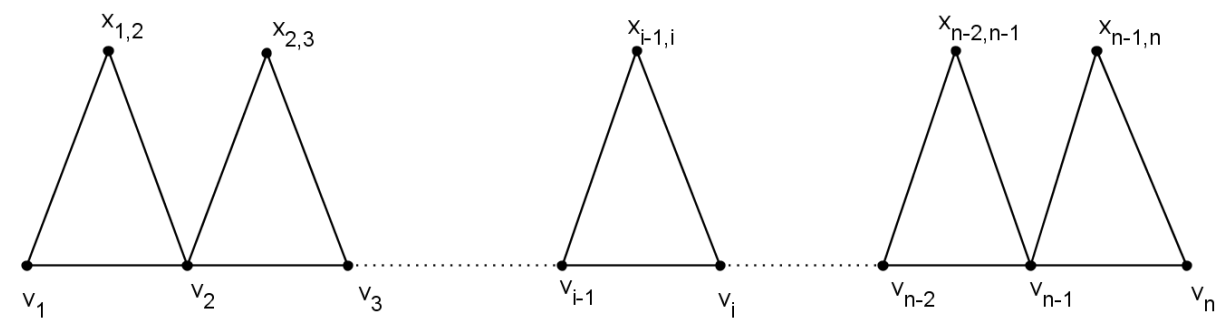

Figure 8. Triangular snake $T_{n}$.

Case 1: $n$ is odd and $n \geq 5$. While finding any $\gamma_{h}$-set of $T_{n}$, the vertices $v_{2}$ and $v_{n-2}$ are taken and the remaining vertices from the subset $S_{k} \subseteq S$ where

$$
S_{k}=\left\{x_{i, i+1} \mid i \equiv 0 \quad(\bmod 2), \quad 2 \leq i \leq(n-3)\right\} .
$$

Clearly, $\left|S_{k}\right|=(n-3) / 2$. Thus,

$$
\gamma_{h}\left(T_{n}\right) \leq 2+\left\lfloor\frac{n-3}{2}\right\rfloor
$$

It is easily seen that

$$
\gamma_{h}\left(T_{n}\right) \geq 2+\left\lfloor\frac{n-3}{2}\right\rfloor
$$

Therefore,

$$
\gamma_{h}\left(T_{n}\right)=2+\left\lfloor\frac{n-3}{2}\right\rfloor .
$$

Case 2: $n$ is even and $n \neq 6$. Note that any common vertex say $x_{i, i+1}$ hop dominates the vertices $v_{i-2}$ and $v_{i}$ of $P_{n}, x_{i-2, i-1}, x_{i+1, i+2}$. Equivalently, the hop degree of any vertex is at most 4 . Hence by choosing vertices from the set

$$
S_{k}^{\prime}=\left\{x_{i, i+1} \mid i \equiv 0 \quad(\bmod 2), \quad 2 \leq i \leq(n-2)\right\} \subseteq S,
$$

any $\gamma_{h}$-set can be obtained which includes the non-hop dominated vertices $v_{2}$ and $v_{n-1}$ too. Thus,

$$
\gamma_{h}\left(T_{n}\right) \leq 2+\left\lfloor\frac{n-2}{2}\right\rfloor .
$$

It is observed that

$$
\gamma_{h}\left(T_{n}\right) \geq 2+\left\lfloor\frac{n-2}{2}\right\rfloor
$$

Therefore,

$$
\gamma_{h}\left(T_{n}\right)=2+\left\lfloor\frac{n-2}{2}\right\rfloor .
$$

Definition 7 [5]. An alternate triangular snake $A T_{n}$ is a graph obtained from the path $P_{n}$, in which every alternate edge of a path is replaced by a cycle $C_{3}$. For example, an alternate double triangular snake is shown in Fig. 9.

An alternate double triangular snake $A D\left(T_{n}\right)$ is obtained from two alternate triangular snakes that share a common path. For example, an alternate double triangular snake is illustrated in Fig. 10.

An alternate triple (four) triangular snakes $A T\left(T_{n}\right)\left(A F\left(T_{n}\right)\right)$ consists of three (four) alternate triangular snakes that share a common path. 


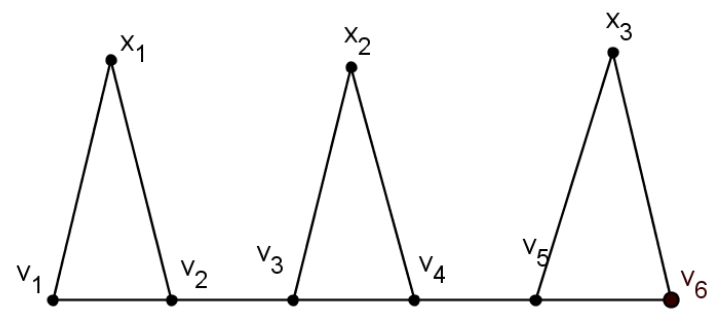

Figure 9. Alternate Triangular snake $A T_{6}$.

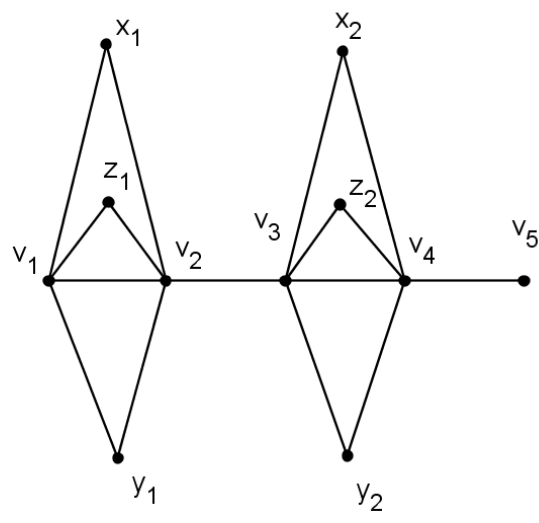

Figure 10. Alternate Triangular snake $A D T_{5}$.

\section{Theorem 8.}

$$
\gamma_{h}\left(A T_{n}\right)=\gamma_{h}\left(A D\left(T_{n}\right)\right)=\gamma_{h}\left(A T\left(T_{n}\right)\right)=\gamma_{h}\left(A F\left(T_{n}\right)\right)=\left\{\begin{array}{lll}
\frac{n}{2}, & \text { if } n \equiv 0,2 \quad(\bmod 4) ; \\
\frac{n+1}{2}, & \text { if } n \equiv 3 \quad(\bmod 4) ; \\
\frac{n-1}{2}, & \text { if } n \equiv 1 \quad(\bmod 4) .
\end{array}\right.
$$

P r o o f. Let us follow the labeling of vertices as described in Theorem 7. Here, $d\left(v_{i}\right)=3$, $i \neq 1, n$ and any vertex of path $P_{n}$ hop dominates at most 3 vertices. In any $\gamma_{h}$-set, it is clear that central vertices of $P_{n}$ alone appear consecutively (see Fig. 11-12).

Case 1: $n$ is even.

Case 1.1: $n \equiv 0(\bmod 4)$. Here, any $\gamma_{h}$-set is of the form

$$
S=\left\{v_{i} \mid i \equiv 2 \quad(\bmod 4), \quad 2 \leq i \leq(n-2)\right\} \cup\left\{v_{j} \mid j \equiv 3 \quad(\bmod 4), \quad 3 \leq j \leq(n-1)\right\} .
$$

Thus, $\gamma_{h}\left(A T_{n}\right) \leq n / 2$. It is easily seen that, $\gamma_{h}\left(A T_{n}\right) \geq n / 2$. Therefore, $\gamma_{h}\left(A T_{n}\right)=n / 2$.

Case 1.2: $n \equiv 2(\bmod 4)$ In this case, $v_{n-2}$ must be chosen in any $\gamma_{h^{-}}$set and the remaining vertices are chosen from $\left\{v_{2}, v_{3}, v_{6}, v_{7}, \ldots v_{n-4}, v_{n-3}\right\}$. Thus, $\gamma_{h}\left(A T_{n}\right) \leq(n-2) / 2+1=n / 2$. It is easily seen that $\gamma_{h}\left(A T_{n}\right) \geq n / 2$. Therefore, $\gamma_{h}\left(A T_{n}\right)=n / 2$. 


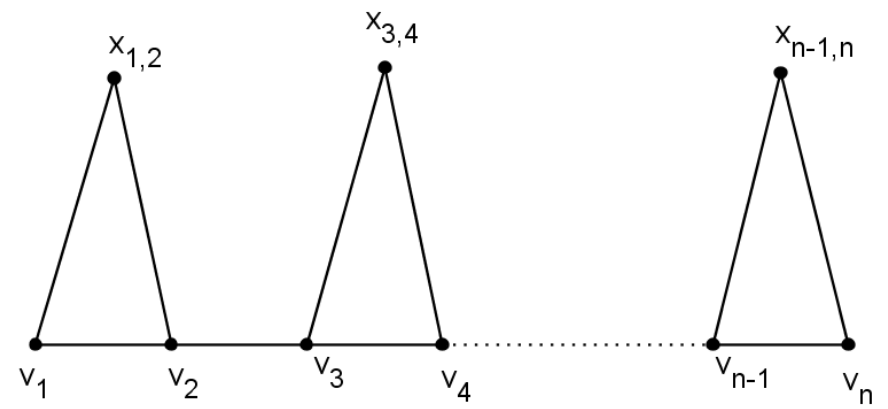

Figure 11. $A T_{n}$, when $n$ is even.
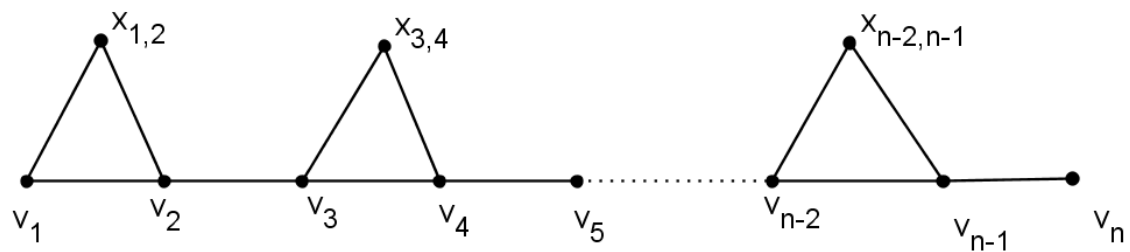

Figure 12. $A T_{n}$, when $n$ is odd.

Case 2: $n$ is odd.

Case 2.1: $n \equiv 1(\bmod 4)$. In this case, any $\gamma_{h}$-set are chosen from $\left\{v_{2}, v_{3}, v_{6}, v_{7}, \ldots v_{n-3}, v_{n-2}\right\}$, with cardinality $(n-1) / 2$. Thus, $\gamma_{h}\left(A T_{n}\right) \leq(n-1) / 2$ and it is easy to verify that $\gamma_{h}\left(A T_{n}\right) \geq(n-1) / 2$. Therefore, $\gamma_{h}\left(A T_{n}\right)=(n-1) / 2$.

Similarly, the case for $n \equiv 3(\bmod 4)$ follows.

Definition 8. A Quadrilateral snake $Q_{n}$ is a graph obtained by replacing each edge of a path $P_{n}$ by a cycle of length 4 .

An alternate quadrilateral snake $A Q_{n}$ is obtained from the path $P_{n}$ by replacing its alternate edges with $C_{4}$.

Proposition 1. (i) $\gamma_{h}\left(Q_{n}\right)= \begin{cases}\frac{n+2}{2}, & \text { if } n \equiv 0,2(\bmod 4) ; \\ \frac{n+3}{2}, & \text { if } n \equiv 1(\bmod 4) ; \\ \frac{n+1}{2}, & \text { if } n \equiv 3(\bmod 4) .\end{cases}$

(ii) $\gamma_{h}\left(A Q_{n}\right)= \begin{cases}\frac{n+1}{2}, & \text { if } n \equiv 1,3(\bmod 4) ; \\ \frac{n+2}{2}, & \text { if } n \equiv 0,2(\bmod 4) \text {. }\end{cases}$

Definition 9. Ciliate is a graph $C(p, s)$ obtained from $p$ disjoint copies of the path $P_{s}$ by linking one end point of each such copy in the cycle $C_{p}$. For example, a Ciliate $C(3,3)$ is shown in Fig. 13. 


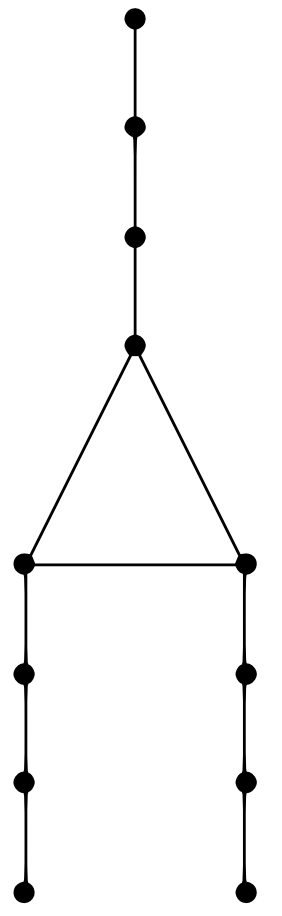

Figure 13. Ciliate $C(3,3)$.

Remark 2. $\gamma_{h}[C(p, q)]=p \gamma_{h}\left(P_{q}\right)$.

Definition 10. A generalized ciliate $G C(p, s, t)$ is obtained by attaching $t$-copies of path $P_{s}$ to each vertex of the cycle $C_{p}$.

Proposition 2. $\gamma_{h}[G C(3, s, t)]=\left\{\begin{array}{cl}2+3 t\left\lfloor\frac{s}{3}\right\rfloor, & \text { if } s \equiv 0(\bmod 6) ; \\ 3+3 t\left\lfloor\frac{s}{3}\right\rfloor, & \text { if } s \equiv 1,3(\bmod 6) ; \\ 3 t\left\lfloor\frac{s}{3}\right\rfloor+1, & \text { if } s \equiv 4,5(\bmod 6) ; \\ 3+3 t\left\lfloor\frac{s}{3}\right\rfloor, & \text { if } s \equiv 2(\bmod 6)\end{array}\right.$

Theorem 9. $\gamma_{h}[G C(4, s, t)]= \begin{cases}2+4 t\left\lfloor\frac{s}{3}\right\rfloor, & \text { if } s \equiv 0,1(\bmod 6) ; \\ 4+4 t\left\lfloor\frac{s}{3}\right\rfloor, & \text { if } s \equiv 2(\bmod 6) \\ 4 t\left\lfloor\frac{s}{3}\right\rfloor, & \text { if } \quad s \equiv 3,4,5(\bmod 6)\end{cases}$

$\mathrm{P}$ r o o f. Let us denote the vertices in the $i^{t h}$ copy of the path $P_{s}$ as $\left\{v_{1}^{i}, v_{2}^{i} \ldots v_{s}^{i}: 1 \leq i \leq t\right\}$. as shown in Fig. 14. Clearly, to hop dominate the leaves and its support vertices in every $i^{\text {th }}$ copy of $P_{s}$, the vertices $v_{s-2}^{i}$ and $v_{s-3}^{i}(1 \leq i \leq t)$ have to be chosen for any $\gamma_{h^{-}}$set of $G C(4, s, t)$.

Case 1: $s \equiv 0,1(\bmod 6)$. To hop dominate $v_{1}$ 's and the vertices of the cycle, any $\gamma_{h}$-set includes $u_{2}, u_{3}$. The remaining vertices in each copy of $P_{s}$ in $\mathrm{GC}(4, \mathrm{~s}, \mathrm{t})$ will induce a path, thus it is sufficient to add to $\left\{v_{s-2}^{i}, v_{s-3}^{i}, v_{s-8}^{i}, v_{s-9}^{i} \ldots v_{5}^{i}, v_{4}^{i}\right\}, 1 \leq i \leq s$ to $\gamma_{h}$-set of $G C(4, s, t)$. Thus, 


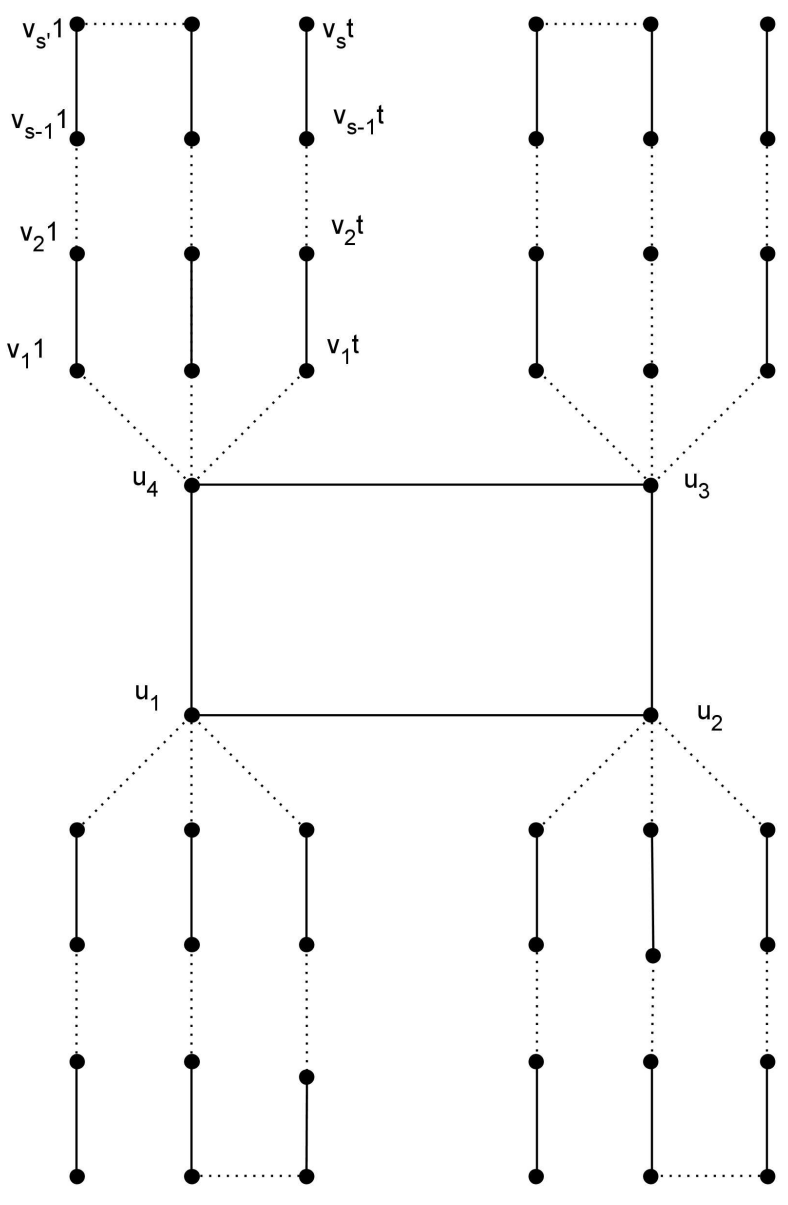

Figure 14. Generalized ciliate $G C(4, s, t)$.

$\gamma_{h}[G C(4, s, t)] \leq 2+4 t \gamma_{h}\left(P_{s}\right) \leq 4 t\lfloor s / 3\rfloor$ and it is easily seen that $\gamma_{h}[G C(4, s, t)] \geq 2+4 t \gamma_{h}\left(P_{s}\right)$. Therefore,

$$
\gamma_{h}[G C(4, s, t)]=2+4 t \gamma_{h}\left(P_{s}\right)
$$

Case 2: $s \equiv 2(\bmod 6)$. Any $\gamma_{h}$-set comprises $u_{1}, u_{2}, u_{3}, u_{4}$ to hop dominate $v_{1}^{i}$ and $v_{2}^{i}$ as well as the vertices of the cycle. Each copy's remaining vertices will induce a path on $(s-2)$ vertices. As a result, $\left\{v_{s-2}^{i}, v_{s-3}^{i}, v_{s-8}^{i}, v_{s-9}^{i} \ldots v_{6}^{i}, v_{5}^{i}\right\}$ are required to form a $\gamma_{h}$-set of $G C(4, s, t)$. Thus, $\gamma_{h}[G C(4, s, t)] \leq 4+4 t\lfloor s / 3\rfloor$ and it is easy to show that $\gamma_{h}[G C(4, s, t)] \geq 4+4 t\lfloor s / 3\rfloor$. Therefore,

$$
\gamma_{h}[G C(4, s, t)]=4+4 t\lfloor s / 3\rfloor .
$$

Case 3: $s \equiv 3,4,5(\bmod 6)$. When $s \equiv 3,5(\bmod 6), H=\left\{v_{s-2}^{i}, v_{s-3}^{i}, v_{s-8}^{i}, v_{s-9}^{i} \ldots v_{3}^{i}, v_{2}^{i}\right\}$ forms a $\gamma_{h}$-set of $G C(4, s, t)$, whereas for $s \equiv 4(\bmod 6), H=\left\{v_{s-2}^{i}, v_{s-3}^{i}, v_{s-8}^{i}, v_{s-9}^{i} \ldots v_{2}^{i}, v_{1}^{i}\right\}$ forms a $\gamma_{h}$-set. Thus, $\gamma_{h}[G C(4, s, t)]=4 t \gamma_{h}\left(P_{s}\right) \leq 4 t(\lfloor s / 3\rfloor+1)$. It is easily seen that $\gamma_{h}[G C(4, s, t)] \geq 4 t(\lfloor s / 3\rfloor+1)$. Therefore,

$$
\gamma_{h}[G C(4, s, t)]=4 t(\lfloor s / 3\rfloor+1)
$$




\section{Conclusion}

In this study, we computed hop domination number for some special families of graphs like triangular, quadrilateral, alternate triangular, alternate quadrilateral snake graphs and examined hop domination number for some generalized graph structures like generalized theta graph, glued path graph. In future, the result obtained for generalized ciliates $p=3,4$ may be extended to $p>4$.

\section{Acknowledgements}

The authors thank the referees for their insightful comments. Also, the authors thank the Department of Science and Technology, Government of India for the financial support to the Department of Mathematics, SASTRA Deemed to be University under FIST Programme Grant No.: SR/FST/MSI-107/2015(c).

\section{REFERENCES}

1. Ayyaswamy S. K., Natarajan C. Hop Domination in Graphs. Manuscript, 2015.

2. Ayyaswamy S.K., Krishnakumari B., Natarajan C., Venkatakrishnan Y.B. Bounds on the hop domination number of a tree. Proc. Math. Sci., 2015. Vol. 125, No. 4. P. 449-455. DOI: $10.1007 / \mathrm{s} 12044-015-0251-6$

3. Balakrishnan R., Ranganathan K. A Textbook of Graph Theory, 2nd ed. NY: Springer, 2012.292 p. DOI: $10.1007 / 978-1-4614-4529-6$

4. Durgun D.D., Lökçü B. Weak and strong domination in thorn graphs. Asian-Eur. J. Math., 2020. Vol. 13, No. 4. Art. no. 2050071. DOI: 10.1142/S1793557120500710

5. Gallian J. A. A dynamic survey of graph Labeling. Electron. J. Combin., 2021. Art. no. DS6. P. 1-576. DOI: $10.37236 / 27$

6. Getchial Pon Packiavathi P., Balamurugan S., Gnanajothi R. B. Hop domination number of caterpillar graphs. Adv. Math. Sci. J., 2020. Vol. 9, No. 5. P. 2739-2748. DOI: 10.37418/amsj.9.5.36

7. Gutman I. Distance in thorny graph. Publ. Inst. Math. (Beograd) (N.S.), 1998. Vol. 63, No. 83. P. 31-36. URL: http://eudml.org/doc/258067

8. Haynes T. W., Hedetniemi S. T., Henning M. A. Topics in Domination in Graphs. Cham: Springer, 2020. 545 p. DOI: $10.1007 / 978-3-030-51117-3$

9. Haynes T. W., Hedetniemi S. T., Slater P. J. Fundamentals of Domination in Graphs. Boca Raton: CRC Press, 1998. 464 p. DOI: 10.1201/9781482246582

10. Haynes T. W., Hedetniemi S. T., Slater P. J. Domination in Graphs-Advanced Topics. Boca Raton: CRC Press, 1998. 520 p. DOI: 10.1201/9781315141428

11. Henning M. A., Rad N. J. On 2-step and hop dominating sets in graphs. Graphs Combin., 2017. Vol. 33. P. 913-927. DOI: 10.1007/s00373-017-1789-0

12. Henning M. A., Pal S., Pradhan D. Algorithm and hardness results on hop domination in graphs. Inform. Process. Lett., 2020. Vol. 153. P. 1-8. DOI: 10.1016/j.ipl.2019.105872

13. Natarajan C., Ayyaswamy S. K. Hop domination in graphs-II. An. St. Univ. Ovidius Constanta, 2015. Vol. 23. No. 2. P. 187-199. DOI: 10.1515/auom-2015-0036

14. Natarajan C., Ayyaswamy S.K., Sathiamoorthy G. A note on hop domination number of some special families of graphs. Int. J. Pure Appl. Math., 2018. Vol. 119. No. 12f. P. 14165-14171. URL: https://www.acadpubl.eu/hub/2018-119-12/articles/6/1314.pdf

15. Pabilona Y. M., Rara H. M. Connected hop domination in graphs under some binary operations. AsianEur. J. Math., 2018. Vol. 11. No. 5 .P. 1-11. DOI: 10.1142/S1793557118500754

16. Rad N. J., Poureidi A. On hop Roman domination in trees. Comm. Combin. Optim., 2019. Vol. 4. No. 2. P. 201-208. DOI: 10.22049/CCO.2019.26469.1116

17. Rakim R. C., Saromines Ch. J. C., Rara H. M. Perfect hop domination in graph. Appl. Math. Sci., 2018. Vol. 12. No. 13. P. 635-649. DOI: 10.12988/ams.2018.8576

18. Salasalan G.P., Canoy S. R., Jr Global hop domination numbers of graphs. Eur. J. Pure Appl. Math., 2021. Vol. 14, No. 1. P. 112-125. DOI: 10.29020/nybg.ejpam.v14i1.3916 
19. Sathiyamoorthy G., Janakiraman T. N. Graceful labeling of generalized theta graphs. Nat. Acad. Sci. Lett., 2018. Vol. 41. No. 2. P. 121-122. DOI: 10.1007/s40009-018-0625-2

20. Shanmugavelan S., Natarajan C. An updated survey on distance-based domination parameters in graphs. Asia Math., 2020. Vol. 4. No. 2. P. $134-149$. URL: http://www.asiamath.org/article/vol4iss2/AM-2008-4207.pdf 